\title{
THE EVOLUTION OF PLANETARY NEBULAE. II. DYNAMICAL EVOLUTION OF ELLIPTICAL PNs AND COLLIMATED OUTFLOWS
}

\author{
Bruce Balick $^{\text {a) }}$ AND Heather L. Preston ${ }^{a)}$ \\ Astronomy Department, University of Washington, Seattle, Washington 98195 \\ VINCENT ICKE \\ Sterrewacht Leiden, Postbus 9513, 2300 RA Leiden, the Netherlands \\ Received 20 August 1987; revised 8 September 1987
}

\begin{abstract}
We present observations mapping the velocity of the gas in $\mathrm{H} \alpha$ and [N $\mathrm{NI}$ ] for the planetary nebulae NGC 40, 2392, 3242, 6543, 6826, 7009, 7354, and 7662. All of these show spatial and kinematic features suggestive of the existence of highly collimated fast outflows, generally characterized by velocities (projected onto the sky) in the range of $10-60 \mathrm{~km} \mathrm{~s}^{-1}$. NGC 2392 is extreme in its outflow velocities of $\sim 170 \mathrm{~km} \mathrm{~s}^{-1}$. To explain the observations, we propose a hydrodynamic mechanism that collimates the gas as it passes outward through a prolate shock front. We adopt the usual fast-wind model, but assume that the slow-wind envelope that surrounds the star is denser near its equator than near the poles. At the onset of the fast-wind stage, this density gradient allows the outer shock to move into the envelope more quickly along the poles than at the equator (i.e., the shock forms as a prolate ellipsoid). A corresponding prolate ellipsoidal reverse shock forms facing the central star. It is this inner shock that begins to collimate the fast wind by deflecting the gas away from the shock normal. Beyond the reverse shock, the high density resulting from the focusing reduces the cooling time of the gas near the collimation axis. Thus, the collimated flow consists of a two-phase gas of cool weakly ionized or neutral knots plowing through the surrounding highly ionized material. The knots, having a larger surface density than their surroundings, act as soft bullets that pierce the outer shock, forming a bow shock. Other warmer material that is less focused about the symmetry axis envelops the knots and enhances the density of the outer shock near the polar caps. A comparison of observed and model isovelocity images shows that this interpretation is tenable for the objects in our sample. In future work, we will test our hypothetical mechanism with additional observations of the kinematic structure of other PNs. We intend to improve the physical motivation and predictive power of our model through the use of two-dimensional numerical hydrodynamical calculations.
\end{abstract}

\section{INTRODUCTION}

The currently popular (but still not critically tested) consensus model of planetary nebulae is one in which a solarmass type star, towards the end of its evolution on the asymptotic giant branch, switches from losing mass in a "slow wind" with a speed of about $10 \mathrm{~km} \mathrm{~s}^{-1}$ and a mass-loss rate of $10^{-4}$ to $-5 \mathscr{M}_{\odot} \mathrm{yr}^{-1}$ to a "fast wind" hurtling out at up to $2000 \mathrm{~km} \mathrm{~s}^{-1}$ and a mass-loss rate of $10^{-7}$ to $-8 \mathscr{M}_{\odot}$ $\mathrm{yr}^{-1}$. In this class of "interacting-winds" models, the fast wind drives a shock wave into the former red giant envelope (RGE) deposited by the slow wind, creating a bright shell within the envelope (Caster, McCray, and Weaver 1975; Weaver et al. 1977; Kwok 1980,1982; Kahn 1983; Lamers 1983; Kwok et al. 1985; Okorokov et al. 1985; Volk and Kwok 1985; Balick 1987a,b).

Balick (1987b, hereafter referred to as Paper I) observed the spatial structure of some 51 planetary nebulae (PNs) in different emission lines and developed a morphological classification scheme consistent with expectations of wind shaping. Here we present detailed kinematic studies of selected members of two of Balick's morphological classes: early- and middle-type ellipticals (hereafter referred to as $\mathrm{eE}$ and $\mathrm{mE}$, respectively). The broad aim of this paper is to establish

a) Visiting Astronomer, Kitt Peak National Observatory, National Optical Astronomy Observatories (NOAO). The NOAO is operated by the Association of Universities for Research in Astronomy, Inc., under contract with the National Science Foundation (NSF). whether interacting winds can explain the morphological and kinematic properties of these two types of nebulae.

The work is presented in four parts. First, we present the global patterns of gas flows in a sample of PNs of similar morphologies (Sec. II). Some of these gas flows turn out to have highly collimated streams in the ends of which lowionization knots are embedded. The kinematics of these flows and their environments are measured by high-dispersion spectroscopic observations taken systematically over each nebula. Second, previously obtained direct CCD images of these PNs are compared with the kinematic information, making it possible for the reader to discern the connection between the kinematics and the morphology. Third, we develop a single, physically motivated model that accounts for the global morphologies, the peculiar small-scale features, and the kinematics of this sample of PNs (Sec. III). Fourth, we endeavor to provide appropriate constraints for future two-dimensional hydrodynamical models of these and other forms of PNs (Sec. IV). Our principal conclusions and directions for future research are summarized in Sec. V.

\section{KINEMATIC PROPERTIES}

\section{a) Observations}

Motions of the nebular gas were studied in the lines of $\mathrm{H} \alpha$ and [N II] $\lambda 6854 \AA$ (hereafter referred to as [N II] for the $\mathrm{eE}$ and ME PNs NGC 40, 2392, 3242, 6543, 6826, 7009, 7354 , and 7662. Nearly all of these PNs show morphological features suggestive of the existence of highly collimated out- 
flows (Paper I). They are found to possess these features in velocity space too. The data were obtained using the echelle spectrograph and CCD detector on the Mayall $4 \mathrm{~m}$ telescope of Kitt Peak National Observatory in April and December, 1986. Except as noted below, the collection and calibration of the data were carried out as described by Balick and Preston (1987). All velocities discussed in this paper are relative to the systemic velocity of the corresponding PN. North, east, south, and west are abbreviated N, E, S, and W, respectively.

The long slit of the echelle spectrograph was placed across the portions of each nebula that were visible on the TV guider at the telescope. Repeated exposures were made as the telescope was offset in a direction perpendicular to the slit. Integration times were the same at all offset slit positions. The details are given in Table I. The slit was 1 " wide for all nebulae except NGC 7009, where a 2" slit was used. The seeing was close to 1.5 at all times, and the skies were generally photometric. Thin clouds (which went unnoticed at the time) affected observations at three slit positions in NGC 2392. After calibration, the data were smoothed to an effective resolution of $8.91 \mathrm{~km} \mathrm{~s}^{-1}$ in velocity and $1^{\prime \prime}$ along the slit. For comparison, the instrumental velocity resolution is $9 \mathrm{~km} \mathrm{~s}^{-1}$, and the thermal broadenings of the $\mathrm{H} \alpha$ and [N II] lines are about 16 and $4 \mathrm{~km} \mathrm{~s}^{-1}$, respectively, for a gas at $\sim 10^{4} \mathrm{~K}$.

The data obtained for some nebulae are very similar in resolution and signal-to-noise ratio to data presented by other workers. We emphasize that the present data differ from most earlier observations in that they were obtained systematically over the bright regions of each object. As described in Balick and Preston (1987), the data can then be presented as a series "isovelocity frames" at velocity offsets of $8.91 \mathrm{~km}$ $\mathrm{s}^{-1}$. Each frame has spatial axes $x$, or position along the slit (pixel size 1" except for NGC 2392, for which a 2" pixel is used), and $y$, or offset position perpendicular to the slit (see Table I for $y$ axis pixel size). This procedure produces frames that suffer a geometric distortion owing to the different pixel sizes in the $x$ and $y$ directions.

The set of isovelocity frames is called a kinematic image. The kinematic images for the various PNs are presented in Figs. 1-8 [Plates 129-136]. The individual isovelocity frames were added to form a total emission line (or "sum") image. These, and the undistorted direct CCD images in the same emission line (Paper I), are also shown in each figure in order that the reader can easily understand the distortions inherent in the isovelocity frames.

\section{b) Nebular Kinematic Features}

In this section we describe the general kinematic properties of each nebula and identify features of particular interest and relevance in subsequent sections. The nomenclature used for morphological features and PN shapes is that of Paper I. It must be emphasized that the discussion below is model independent.

NGC 40. The morphology and ionization of this very interesting nebula have been discussed by Balick et al. (1987), Jacoby, Quigley, and Africano (1987), Sabbadin and Hamzaoglu (1982), and others. Using the morphological designations of Paper I, NGC 40 is the latest type of any of the elliptical PNs discussed in the present paper. A very bright elliptical rim which is incomplete along the major axis of the nebula is the most conspicuous feature. A faint inner halo lies just outside the rim (Paper I). Filamentary nebulosity is found along the major axis throughout the nebula, as shown in the direct CCD image of Fig. 1.

We present just the kinematic image in the [N II] line (the $\mathrm{H} \alpha$ data are essentially identical, except that the $\mathrm{H} \alpha$ line velocity resolution is degraded by the relatively large thermal broadening in this line). The inner regions of the nebula, consisting of the bright rims and the material between them, show a very obvious pattern of expansion in the series of isovelocity frames. The pattern of motions in the inner region can be interpreted as the expansion of a hollow prolate elliptical bubble whose ends are severed. The bubble expansion velocity is $\sim 25 \mathrm{~km} \mathrm{~s}^{-1}$. Smaller expanding bubbles are attached to the open ends of the inner expanding prolate ellipsoid.

NGC 2392. Only the [ $\mathrm{N} \mathrm{II}$ ] kinematic image is presented (Fig. 2) for the reason mentioned a paragraph above. NGC 2392 exhibits extremely complex patterns of motions throughout the kinematic image. Forty-two isovelocity frames are shown in Fig. 2, which span velocities from -191 to $+191 \mathrm{~km} \mathrm{~s}^{-1}$, and nebulosity is visible in every frame. However, all of the features at negative velocity have extremely similar positive-velocity counterparts on opposite sides of the central star, except for the features at zero velocity. For simplicity, we shall describe primarily the positivevelocity features.

"Spokes" are radial features seen in a zone between 12 " and 24 " from the nucleus and only near zero velocity (frames 2-23). Inside the spokes is a bright circular "rim" of radius $\sim 7$ " centered on the nucleus. As the velocities increase over 15 frames $\left(4.5\right.$ to $\left.140 \mathrm{~km} \mathrm{~s}^{-1}\right)$, the rim shrinks in

TABLE I. Description of kinematic images.

\begin{tabular}{|c|c|c|c|c|}
\hline Nebula & $\begin{array}{c}\text { Slit } \\
\text { P.A. }\left(^{\circ}\right)\end{array}$ & $\begin{array}{l}\text { No. Slit } \\
\text { Positions }\end{array}$ & $\begin{array}{c}\text { Integ. Time } \\
\text { per Exposure(s) }\end{array}$ & $\begin{array}{c}\text { Rel. Slit } \\
\text { Spacing (") }\end{array}$ \\
\hline NGC 40 & 0 & 11 & 400 & 4 \\
\hline 2392 & 90 & 25 & 300 & 2 \\
\hline 3242 & -30 & 13 & 200 & 2 \\
\hline 6543 & 0 & 9 & 150 & 2 \\
\hline 6826 & 0 & 15 & 200 & 3 \\
\hline 7009 & 77 & 7 & 200 & 2 \\
\hline 7354 & 30 & 9 & 200 & 2 \\
\hline 7662 & 0 & 7 & 300 & 3 \\
\hline
\end{tabular}


size to $\sim 1.5$, and its center moves northward by about $3 "$. This pattern of motions has been interpreted as a uniformly expanding, elongated, ellipsoidal shell whose major axis lies near the line of sight (Gieseking, Becker, and Solf 1985hereafter referred to as GBS; O'Dell and Ball 1985; Reay, Atherton, and Taylor 1983). The latter authors also identified the bright, amorphous path of emission seen about 10 " $\mathrm{N}$ of the nucleus in frames at velocities between about 30 and $80 \mathrm{~km} \mathrm{~s}^{-1}$, and its even brighter negative-velocity counterpart, as "fur."

O'Dell and Ball were the first to observe the gas at the highest velocities (frames 1-5 and 38-42), which they described as jet-type bipolar outflows. We prefer a characterization as high-velocity knots, though either description is apt. Whether the high-velocity outflowing material is a separate physical entity from, or some sort of extension (with changing position angle) of, the expanding ellipsoidal shell is unclear. In the latter case, the outflowing velocity vector must swing in position angle by about $60^{\circ}$ between velocities of 140 and $160 \mathrm{~km} \mathrm{~s}^{-1}$. Such behavior is not without precedent (Balick and Preston 1987). It is curious, and possibly coincidental, that the high-velocity gas projects onto the largest gaps between the spokes at the same radius.

NGC 3242. As seen in $\mathrm{H} \alpha$, this $\mathrm{PN}$ is the archetypical eE PN. Morphologically, NGC 3242 shows a nearly circular bright rim, outside of which is a smooth halo with a sharp outer boundary. The rim of this nebula (like the rim of NGC 7662 ) is also conspicuous in emission lines of $\mathrm{He}^{++}, \mathrm{O}^{++}$, $\mathrm{H}^{+}, \mathrm{N}^{+}$, and $\mathrm{O}^{0}$ (Paper I).

Two conspicuous knots of very low ionization (and without any $\mathrm{H} \alpha$ counterpart) lie outside the rim, along both extensions of its major axis. (The knots are also detectable in the [O I] $\lambda 6300 \AA$ line.) Similar knots are found in NGC 6826 and 7662. Between the rim and the knots lie triangular (conical?) bridges of emission seen in $\mathrm{H} \alpha$ and [O III] $\lambda 5007$ $\AA$. The knots and the bridge that joins them to the rim are called "ansae" in Paper I. Although the inner halo is smooth in appearance, its emission measure is largest along the $m i$ nor axis of the rim.

The kinematic images in Fig. 3 show very clearly that the [N II] knots and the bridges have anomalous velocities relative to the $\mathrm{H} \alpha$ observed in the same directions. The observed velocity differences range up to $25 \mathrm{~km} \mathrm{~s}^{-1}$. This originally led Weedman (1968) to propose a two-shell kinematic system, but such a model is not supported by the present data. The true velocity of the knots relative to the nebula will be larger owing to projection effects. Velocities seem to increase along the length of the ansae behind the knots.

As in the case of NGC 40 already discussed, the kinematics of the rims suggest that they are the boundary of an expanding bubble of nearly circular outline on the sky. The bubble expansion velocity appears to be about $25-30 \mathrm{~km} \mathrm{~s}^{-1}$. Systematic motions in the halo appear to be less than the thermal width of the $\mathrm{H} \alpha$ line. The inner halo is too faint to be studied in the present [N II] kinematic image. Future [O III] kinematic images might reveal important kinematic structure in the halo.

NGC 6543. The kinematics of this nebula have been discussed extensively elsewhere (Balick and Preston 1987, and references therein). The data for this nebula have been included here for comparison to other PNs. NGC 6543 shows clear evidence of a bipolar outflow, both morphologically (the prominent [N II] "tails") and kinematically (Fig. 4) (also called "jets" by Solf (1986)). The bipolar tails are visible in $\mathrm{H} \alpha$ and [ $\mathrm{N}$ II] out to velocities of $\pm 50 \mathrm{~km} \mathrm{~s}^{-1}$. The tails grow fainter and twist clockwise with increasing distance from the body of the nebula, as though they were counterrotating. Prominent in the isovelocity frames around zero velocity are the "caps"-high-surface-brightness features at the northern and southern extremities of the nebula-which separate the body of the nebula from the tails. The caps have projected velocities close to zero.

NGC 6826. This PN and NGC 3242 are kindred spirits, and most of the discussion of NGC 3242 applies to both objects. NGC 7662 shares many similarities also. The ansae, low-ionization knots, rims, and inner halos (with highest emission measure along the minor axis of the elliptical rim) shown here are present in too many of the sample PNs to be simply dismissed as anomalies.

The ansae and knots are visible to velocities of $\pm 53.4 \mathrm{~km}$ $\mathrm{s}^{-1}$ in the $\mathrm{H} \alpha$ line and $\pm 44.5 \mathrm{~km} \mathrm{~s}^{-1}$ in the [N II] line (Fig. $5)$. This curious circumstance may imply that the ansae are being decelerated at their leading edges (since the [N $\mathrm{II}]$ is clearly outside of the $\mathrm{H} \alpha$ in position). More likely, the persistence of the knots at large $\mathrm{H} \alpha$ velocities could be caused by the better signal-to-noise ratio, or the larger intrinsic width of the $\mathrm{H} \alpha$ line relative to [ $\mathrm{N}$ II]. These ansae are also quite prominent in [O II] $\lambda 3727 \AA$ (Phillips and Reay 1983), but not in the high-excitation line of [Ne III] $\lambda 3868 \AA$.

The bright elliptical rim shows only subtle evidence of systematic expansion, unlike NGC 3242 and 7662. If the rim is the shell of an expanding ellipsoid, then the major axis of the ellipsoid lies close to the plane of the sky. Also unlike NGC 3242, the inner halo of NGC 6826 shows systematic internal motions (all between about -20 and $+20 \mathrm{~km}$ $\mathrm{s}^{-1}$ ) superimposed on a quiescent background. There appears to be a small velocity gradient in the halo from NE to SW. If real, the inner halo may be rotating slowly. It should be noted that the central star of this nebula has been described as a binary (Kolesnik and Pilyugin 1986).

NGC 7009. This nebula is one of the archetypes of the spindle-shaped morphological class (Reay and Atherton 1985). In Paper I the morphology is described as $\mathrm{mE}$, although a designation of e-mE might be more appropriate. The sequence of [ $\mathrm{N} \mathrm{II}$ ] isovelocity frames of NGC 7009 is of the same general appearance as NGC 3242, NGC 6826, NGC 7354, and NGC 7662. (The $\mathrm{H} \alpha$ kinematic image, though brighter, shows little kinematic structure, owing to the large thermal broadening of this line. The interested reader should consult Reay and Atherton.)

NGC 7009 differs from other PNs studied here in that its ansae project far outside the main body of the nebula. There is a bright and highly elongated elliptical rim in P.A. $\sim 77^{\circ}$. Near opposite ends of the rim are two caps seen only in [N II] and [O I] (Paper I), much like the caps in NGC 6543 discussed by Balick and Preston (1987). However, the caps in NGC 6543 have negligible projected velocities, but those in NGC 7009 have equal and opposite velocities of about $\pm 40 \mathrm{~km} \mathrm{~s}^{-1}$ (Fig. 6). The sense of the velocity gradient of the caps is opposite to that of the ansae and the elliptical rim, as first noted by Reay and Atherton. The situation is most puzzling and difficult to interpret.

NGC 7354. This is a classical $\mathrm{mE}$ PN. The $\mathrm{H} \alpha$ image (Fig. 7) shows an elliptical bright rim which, like NGC 40, is open at each end. An inner halo lies just beyond the rims. Like other PNs, the emission measure of the halo is largest along the minor axis of the elliptical rim (and nearly undetectable along the major axis in this case). The [N II] image is exceedingly faint $\left(\mathrm{N}^{+}\right.$is a highly marginal ionization state). Although the outlines of NGC 7354 appear the same in both 
$\mathrm{H} \alpha$ and [ $\mathrm{N}$ II], the bright rim appears only in lines emitted by highly ionized species such as $\mathrm{He}^{++}, \mathrm{O}^{++}$, and $\mathrm{H}^{+}(\mathrm{Pa}-$ per I). Two bright [N II] patches project onto the rim-halo interface. Most intriguingly, there is a faint pair of linear "antennae" seen in the [N II] line that protrude nearly radially from both extremities of the bright elliptical rim.

The $\mathrm{H} \alpha$ kinematic image is qualitatively the same as that of NGC 40. The elliptical rim can be identified with an expanding ellipsoid. The central ellipsoid is open at the poles, from where two roughly conical features extend along the symmetry axis (we later identify these features with bow shocks extending behind the low-ionization knots; they play a key role in the models to be discussed below). The two [N II] patches seem to have $\mathrm{H} \alpha$ counterparts in the kinematic images. The kinematics of the patches suggest that they lie at the interface of the ellipsoid and the inner halo. Unfortunately, the [ $\mathrm{N}$ II] antennae are too faint to be detected on the echelle spectrograph even during a long (600 s) integration through a wide slit.

NGC 7662. Like NGC 3242 and 6826, NGC 7662 is an eE PN with a bright elliptical rim, inner halo, and low-ionization knots. As in the case of NGC 3242, the bright rim is very conspicuous in lines of $\mathrm{He}^{++}, \mathrm{O}^{++}, \mathrm{H}^{+}, \mathrm{N}^{+}$, and $\mathrm{O}^{0}(\mathrm{~Pa}-$ per I). The inner halo has its highest emission measure along the minor axis. Unlike NGC 3242 and 6826, the low-ionization knots of NGC 7662 lie near the outer edge of the nebula at various position angles, reminiscent of NGC 2392.

The kinematic images (Fig. 8) have three main components. First is the bright elliptical rim which, like the rim in NGC 2392, shrinks in diameter and moves increasingly and symmetrically off center as velocities diverge from zero. This is the pattern expected for a prolate and uniformly expanding ellipsoid with a maximum projected expansion velocity of $\pm 60 \mathrm{~km} \mathrm{~s}^{-1}$ along its major axis (the true space velocity is certainly much larger because of projection effects).

Second, the inner halo is nearly featureless, with exceptions discussed later. Most of the bright [ $\mathrm{N}$ II] knots seen along the nebular perimeter have small projected velocities. Like NGC 6826, outside the inner halo is another halo characterized by even smaller velocities.

Third, there is a pair of high-velocity features on opposite sides of the inner halo close to the major axis of the bright rim. These features seem to connect to the expanding ellipsoid at its extreme ends, especially to the SSW. The NNE feature is best described as a knot, and the SSW feature as a "jet." Both features are especially clear in the [N II] line and are characterized by projected outflow velocities between \pm 40 and $60 \mathrm{~km} \mathrm{~s}^{-1}$. The correction for projection will be large-easily a factor of 2 . The SSW jet shows a complex pattern of velocity changes along its length, as can be easily discerned in the [N II] kinematic image. It is possible to speculate that if viewed along an axis close to the collimated outflow, the kinematics of NGC 7662 would closely resemble those of NGC 2392 in most respects.

Finally, we note that there are some highly systematic motions observable in faint features in the inner halo. One arclike feature is especially conspicuous in frames 10-13 stretching through the nebula in position angles between $-10^{\circ}$ and $120^{\circ}$. There appears to be a faint negative-velocity counterpart in frames 3-6.

\section{HYDRODYNAMIC MODELS}

a) One-Dimensional Wind-Driven Models

It is increasingly accepted that the morphology of bright rim plus surrounding amorphous inner halo seen in many planetary nebulae arises as a consequence of the interaction between a stellar wind and the remnant of an older red giant envelope (RGE). The envelope was presumably deposited during a mass-loss phase when the progenitor star was on the asymptotic giant branch. At some point, the star switches from a comparatively dense slow wind $\left(\sim 10 \mathrm{~km} \mathrm{~s}^{-1}\right)$ to one that is tenuous and very fast (up to $2000 \mathrm{~km} \mathrm{~s}^{-1}$ ). Although the momentum densities in these two wind phases are similar at first, the velocity difference implies that once the very supersonic wind begins, it will drive a shock wave into the slower RGE. A reflection shock then propagates upstream into the fast wind. Models that show the evolution of such a system in one dimension have been discussed by Castor, McCray, and Weaver (1975), Weaver et al. (1977), Kwok (1982), Lamers (1983), and Kahn (1983).

\section{b) Nonspherical Wind-Driven Models \\ 1) Overview}

The basic features of the wind-driven bubble models agree with the observations of some early-type circular PNs, although the inner shock is probably too close to the central star to be readily observable (e.g., Kahn 1983; Okorokov et al. 1985). However, it has become apparent that there is a large subclass of planetaries showing features that consistently indicate a need for modification of such models. These features are the ones we have emphasized in Sec. II, namely ellipsoidal inner rims, pairs of knots (ansae) protruding from the sharp ends of these ellipses, and occasional bright polar caps (Mathews 1978; Balick 1987b).

We believe that all these remarkable features are due to one underlying cause, namely a global inhomogeneity of the RGE into which the fast wind propagates. It can be readily demonstrated that an equatorial density excess leads to the formation of shocks that have cross sections ranging from prolate-spheroidal to hourglass shapes (Kahn and West 1985; Icke 1987). It is less obvious that the ansae and the polar caps are a consequence of the same mechanism, because it would seem that the highly anisotropic morphology that we observe in the kinematic images requires a much more strictly collimated outflow.

However, the reverse shock is also expected to be at least temporarily elongated, roughly in the same direction as the outer shock (Icke 1987). Hence, when the stellar wind passes the reverse shock, it is deflected away from the shock normal in the usual way; in the present configuration, this leads to a focusing of the stellar wind along the symmetry axis of the outer envelope (see Fig. 9). It has been found that the gas is most effectively focused if the axial ratio of the inner ellipsoidal shock is a comparatively modest 0.6 (Icke 1987). Once the gas is forced to converge towards the axis, its higher density increases both the cooling and recombination rates, dropping its ionization and temperature below that of the surrounding gas. Thus, the gas that is deflected towards the axis of the ellipsoid is likely to cool and recombine, producing a small component, or "knot," within the $\mathrm{PN}$ that is reminiscent of the cool phase of the interstellar medium, surrounded by the hot phase of the bulk of the nebula. Cooling by dust in the dense knots can accelerate the drop in temperature even more. Thus, we expect that the low-ionization knots, or soft bullets, move radially along the symmetry axis.

Because of their high density, these knots are not rapidly decelerated, and move essentially with the speed of the gas as it leaves the reverse shock. This speed is still faster than that 


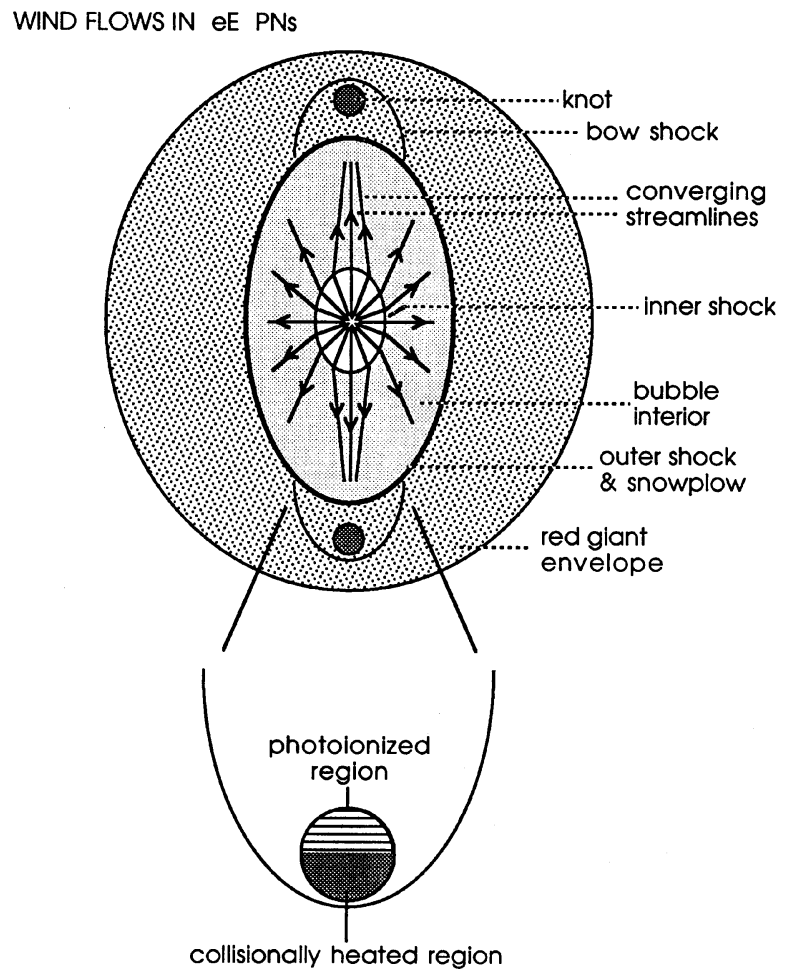

FIG. 9. Sketch of the spindle model. Top: the relative locations of the RGE and the central star, the latter surrounded by its reverse shock and the outer shock. Bottom: a knot that has formed in the collimated axial flow, preceded by its bow shock. The upstream side of the knot is collisionally heated: possibly the downstream side shows some photoionization.

of the outer shock, which is therefore ultimately overtaken by the collimated flow. When the knots break through the outer shock, they may deposit "debris"- - entrained gas, outer ablation layer-which might be observed as bright enhancements, especially in proximity to the knots.

A further disturbance of the polar region may be caused by the gas that is expected to surround the knots. When the gas that is focused along the axis cools, it passes through the temperature region in which the transition occurs that is responsible for the occurrence of two phases in the interstellar medium (Field, Goldsmith, and Habing 1969). Thus, the axial gas consists of a cool neutral flow that is well collimated, surrounded by a warmer "placental" medium with less collimation. This material, although not as cool and dense as the axial knots themselves, is yet sufficiently collimated to impinge preferentially on the poles of the RGE. The cool knots have a high surface density, so that they can breach the outer shock, whereas the warm placental gas represents a mild density enhancement nearby. Where placental gas collides with the RGE, bright collisionally heated polar caps might be expected to form.

\section{2) Plausibility}

Inspection of the spindle-like shapes of early- and middletype elliptical PNs readily shows that the requisite asphericity in the density of the RGE is ubiquitous. The direct CCD images of the inner halo of every elliptical PN in this paper show an enhancement of emission measure along their short axes. The single exception is NGC 2392, which is probably highly inclined (O'Dell and Ball 1985).
Consider the early-type PN NGC 7662, in which a flattening $b / a=0.85$ is measured. It is interesting to note that this value corresponds nicely to the observed difference between the temperature in the polar and in the equatorial regions (Kupferman 1983), in the following sense: If the pressure gradient at the base of the fast wind is spherically symmetric (as seems plausible for a compact star like a white dwarf), we expect that the momentum density and the pressure distribution in the envelope will have the same symmetry. If then the equatorial density is higher (for example, because of rotation of the parent star-see, for example, Friend and Abbott 1986-though we must stress that the cause of the asymmetry is conjecture), the equatorial speed must be smaller in the same proportion.

Thus, the axial ratio should equal the density ratio, which - because of pressure balance in an ideal gas-should equal the temperature ratio. From Kupferman's (1983) data on the temperatures, we calculate an expected value of $b / a=0.83$, in agreement with the value measured from our images. Since the ellipticity of the reverse shock is expected to be larger than that of the outer one, considerable focusing is expected to occur. NGC 3242, 6826, and perhaps the highly inclined PN NGC 2392 all seem to be similar to NGC 7662 , though not as intensively studied observationally.

\section{c) Modeling Morphological and Kinematic Features}

Having now argued that there is reasonable cause to believe that the general morphological and kinematic features of PNs have a hydrodynamic origin, a more quantitative description of the process is undertaken.

The overall appearance we expect on the basis of the above interpretation has been sketched in Fig. 9 (see also the figures in Icke 1987, and Fig. 15 in Paper I). Characteristic features are (1) the equatorial density enhancement in the RGE, which is the root cause of what follows; (2) the prolate ellipsoidal outer shock (previously called an ellipsoidal rim), moving into the envelope at modest speed $\left(20 \mathrm{~km} \mathrm{~s}^{-1}\right.$ or so) and Mach number (about 2); (3) the prolate ellipsoidal reverse shock, which is probably difficult to observe; (4) the stellar fast wind, focused by passage through the reverse shock; (5) the cooling stream along the symmetry axis; (6) cool, neutral knots at the leading edge of the axial streams, moving rather faster than the outer shock (on the order of 60 $\mathrm{km} \mathrm{s}^{-1}$ ); (7) a bow shock preceding each knot, with a Mach cone connecting rearwards with the outer ellipsoidal shock, in which collisional heating takes place.

The goal is to generate a set of model kinematic images for comparison to the observations. We assume that the model is stationary. Consider a spherically expanding shell, pierced on two opposing sides by hyperboloidal bow shocks. A hyperbola is usually a good approximation to such a shock (Fig. 10; cf. the illustrations in Van Dyke 1982). Let the opening half-angle be $A$; then, the equations for the shape and the slope of the bow shock are

$$
\begin{aligned}
& r=\sqrt{z^{2}-a^{2}} \tan A, \\
& \tan \phi=\frac{d r}{d z}=\frac{z}{\sqrt{z^{2}-a^{2}}} \tan A,
\end{aligned}
$$

where $z$ is the axial coordinate along the symmetry axis, $r$ is the radial coordinate perpendicular thereto, $a$ is the semimajor axis of the hyperbola, and $\phi$ is the angle between the shock and the axis. In the frame comoving with the shock, the external gas flows in parallel to the axis. Upon passing 


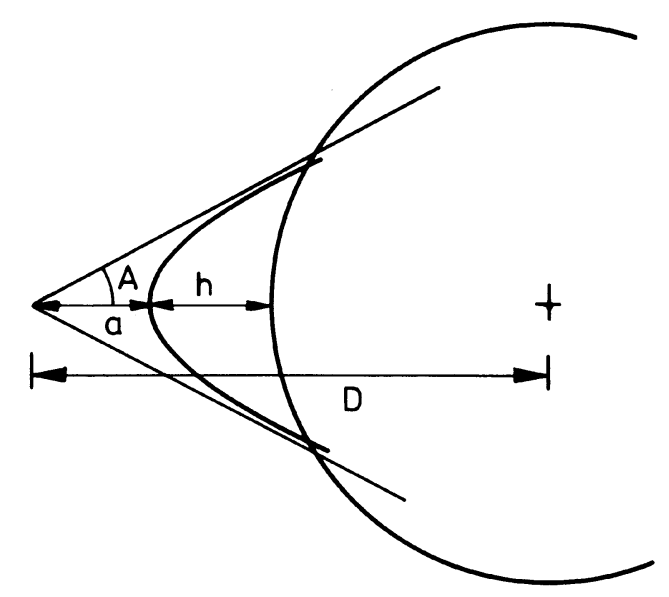

FIG. 10. Geometrical quantities in kinematical model of E-type planetary nebulae. The quantities $h, D$, and $A$ can be varied independently, provided that the hyperbola has an intersection with the sphere (this requires $D<\sin A$ ).

the shock, the flow is deflected by an angle $\chi$, which obeys the well-known equation

$$
\operatorname{cotan} \chi=\tan \phi\left[\frac{(\gamma+1) M^{2}}{2\left(M^{2} \sin ^{2} \phi-1\right)}-1\right]
$$

(e.g., Landau and Lifschitz 1959, p. 333), where $\gamma$ is the adiabatic index of the gas (which we will take to be $5 / 3$ ), and $M$ is the Mach number of the knot that causes the bow shock:

$$
M=w / s .
$$

Here $w$ is the speed of the knot along the symmetry axis and $s$ is the sound speed in the RGE. The Mach number is connected with the asymptotic angle of the shock wave through the expression

$$
\sin A=1 / M
$$

(e.g., Courant and Friedrichs 1976, p. 262).

We now proceed as follows. We prescribe $a$ in Eqs. (1) and (2), and the external Mach number $M$. Then Eq. (5) yields $A$, and we can determine the shape of the bow shock. The velocity deflection $\chi$ is then obtained from Eq. (3), and the postshock speed $v$ becomes

$$
v=w \frac{\sin \phi}{\sin (\phi-\chi)}\left[\frac{\gamma-1}{\gamma+1}+\frac{2}{(\gamma+1) M^{2} \sin ^{2} \phi}\right]
$$

(Landau and Lifschitz 1959, p. 334). In the observer's frame, the postshock velocity vector is then

$$
\left(u_{r}, u_{z}\right)=(v \sin \chi, v \cos \chi-w) \text {. }
$$

This expression describes the cap velocity ahead of the point where it joins the expanding sphere (this point can be found algebraically, once the distance $D$ between the center of the sphere and the cap apex has been given, together with the height $h$ of the cap itself). To obtain the net velocity of the whole spindle model, we add a spherically symmetric component representing the expansion of the central bubble (Fig. 10).

Simulated kinematic images obtained with this model are shown in Figs. 11-13. We have chosen units such that the radius and the expansion speed of the sphere are unity. The frame velocity widths and spacings are representative of those used in our observations. On the basis of a series of such models, we find the following general trends:
(1) The overall morphology of the isovelocity frame sequences is strikingly similar to those observed, allowing the conclusion that the wind-driven spindle model is tenable.

(2) As one proceeds from the central velocity frame to larger radial velocities, the tip of the bow shock shows up as a detached patch. The manner in which this detachment occurs gives good information about the orientation of the spindle axis with respect to the line of sight.

(3) The number of frames in which one sees a patch without a trace of the central sphere is a good indicator of the relative velocity difference between the sphere and the bow shock. Because the present observations show that the number of such shell-less frames is typically only one or two (cf. NGC 6826 and 7662), it appears that the bow shock does not move faster than about 1.2-1.5 times the speed of the spherical shock.

(4) Models like these place virtually no restrictions on the external Mach number. As can be inferred from Eq. (3), and as can be seen in the intermediate frames of Figs. 11-13, the postshock velocity changes rapidly near the very tip of the bow shock, but the approach of the shock to the Mach angle is masked by the connection with the spherical shell. The best estimator of the Mach angle is simply the opening angle of the spindle tip, but this is subject to projection effects; moreover, the brightness of the downstream parts of the bow shock is found to be small.

\section{d) Comparison with Specific Nebulae}

NGC 40 and 7354. The central "empty" regions in the zero-velocity isovelocity frames (hereafter referred to as the zero frame) of these nebulae show clearly that the inner sphere and the inner parts of the ansae are connected. As can be seen from Fig. 11, this is characteristic of spindles with a very small inclination to the line of sight. This effect, plus the evident symmetry of the observed isovelocity frames with respect to reflection about the equator, leads us to conclude that the axis of inclination lies within $10^{\circ}$ of the plane of the sky.

NGC 2392. The Eskimo Nebula is an egregious example of a spindle-type nebula because it has a circular appearance. However, close inspection of the kinematic image shows that the ansae first appear as knots in the high-velocity frames. Proceeding towards lower velocities, these features are seen to broaden and form a ring, which subsequently encompasses the central star, with which it is essentially concentric at zero velocity. This is precisely the behavior seen in Fig. 13, and is typical of shells pierced by a bow shock seen nearly end-on. Our models indicate an inclination of some $70^{\circ}$. This is in good agreement with the findings of Weedman (1968) and of O'Dell and Ball (1985).

NGC 3242. The inner parts of the ansae are practically entirely filled in the [N II] kinematic image; furthermore, the ansae are clearly present even in the zero frame. Comparison with the models of Figs. 11-13 shows that this implies an intermediate orientation of the spindle; we find that an inclination of $30^{\circ}$ is about right. The electron temperature has been mapped across the face of this nebula, and is found to be lower at the poles (Reay and Worswick 1982), in keeping with the higher cooling rate expected for the focused axial flow. The ansae are seen to cross the position of the ellipsoidal shock (cf. Fig. 12), indicating the presence of a velocity field that is not a simple continuation of the central sphere, in agreement with our model. In the direct [N II] CCD image, it is clear that the ansae are not exactly collin- 

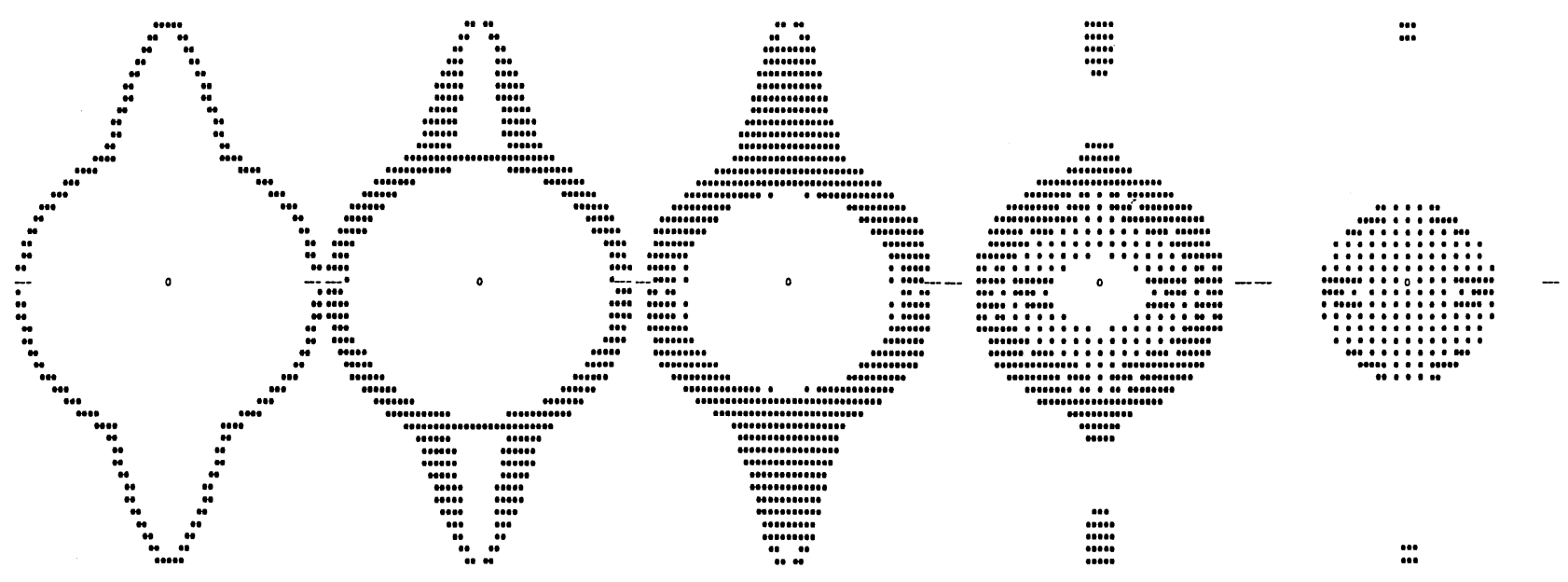

FIG. 11. Isovelocity frames of a model spindle with $M=4, D=2.8, h=0.8, w=3$, zero inclination, and channel width 0.2 . From left to right, the channel centers are $0,0.25,0.5,0.75,1.0$.

ear. This behavior is natural in the shock-focusing model, because the reverse shock cannot be expected to be an exact spheroid, but it would seem to contradict "jet" models wherein the collimation is achieved by means of a dense, small circumstellar disk.

NGC 6862. The inner ring is very bright in $\mathrm{H} \alpha$ and faint in [N II], whereas the reverse is true of the knots. Therefore, the comparison with the model maps is less straightforward. Taken together (i.e., ignoring the excitation differences), the observations appear to indicate another spindle with intermediate inclination, probably between $20^{\circ}$ and $40^{\circ}$. Weedman (1968) found a $40^{\circ}$ inclination angle.

NGC 7662. The $\mathrm{H} \alpha$ and [N II] kinematic images are generally similar in appearance to those in NGC 2392, 3242, and 6826 . The derived inclination is about $40^{\circ}$. The outer edge of the envelope is much more pronounced in NGC 7662 than in most PNs, especially in the [ $\left.\mathrm{N}_{\mathrm{II}}\right]$ images, leading to possible confusion in the interpretation. However, comparison with Fig. 12 shows that the inner small ellipse and the ansae behave as expected.

\section{e) Effect of Nonzero Linewidths}

The interpretation of the position-velocity data cube is evidently limited by the effects of finite resolution and limited surface-brightness sensitivity. Lack of sensitivity is a bit of a problem when trying to discern the downstream parts of the bow shock; here the flow begins to approximate the Mach angle, so that the shock becomes quite weak (e.g., Landau and Lifschitz 1959, p. 457ff). Note that $\chi$ approaches zero as $\phi$ approaches $A$ in Eq. (3).

More insidious is the problem of finite spectral resolution. We have investigated the influence of finite frame bandwidth by taking a typical frame of a model nebula of intermediate inclination, and splitting it into successive frames of decreasing width (Fig. 14). The full width of the first slice was 0.8 , centered on observed velocity $v=0.75$. A fairly featureless picture, only the outline of which reminds one of the presence of collimated flow, results. Next, the slice was split into two, centered on $v=0.95$ and 0.55 , having a width of 0.4 .
This produces a dramatic improvement in one's ability to discern the characteristic appearance of the pierced shell. Another step in resolution, to width 0.2, gives some more improvement, but beyond that, little more is gained. We conclude that frame widths of about $15 \%$ of the shell speed are optimal, unless one were awash in observing time. In the present study, characteristic frame widths are in the vicinity of 0.25 to 0.4 .

Very high-spectral-resolution observations are not of much use if the intrinsic width of the observed line is large. The effects of linewidth are roughly similar to those of wide frames. An example can be seen in NGC 6826 and possibly in NGC 7662, where the $\mathrm{H} \alpha$ images (thermal linewidth $\sim 16 \mathrm{~km} \mathrm{~s}^{-1}$ ) of the ansae continue to higher-velocity extremes than the corresponding [N II] (thermal linewidth $\sim 4 \mathrm{~km} \mathrm{~s}^{-1}$ ). It is clearly preferable to use lines of massive ionic species such as $\mathrm{O}^{++}$to map the nebular kinematics rather than $\mathrm{H} \alpha$. (Note that the [O III] $\lambda 5007 \AA$ direct images of most PNs shown in Paper I appear virtually identical to those of $\mathrm{H} \alpha$ ). Kinematic studies in [O III] amd [N II] lines are especially interesting in that they sample complementary nebular volumes with essentially the same intrinsic linewidth.

\section{f) Comparing Model and Observed Brightness Distributions}

In constructing our model kinematic images, we have made only a rudimentary effort at simulating the brightness distribution. Figures 11-14 were obtained by covering the surface of the spindle with a net of points that were equally spaced along the spindle axis and in azimuthal angle. This produces a surface density of points that is biased towards the ansae, in accordance with the general impression of spindle-shaped planetaries. However, a more precise representation could obviously be obtained only by a fairly detailed calculation of the radiative-exchange processes and the consequent line strengths in the nebulae. This is well beyond the scope of this paper. Moreover, the emissivity of the bow shock depends sensitively on the effective strength of the shock (that is to say, taking the shock obliquity into account). 


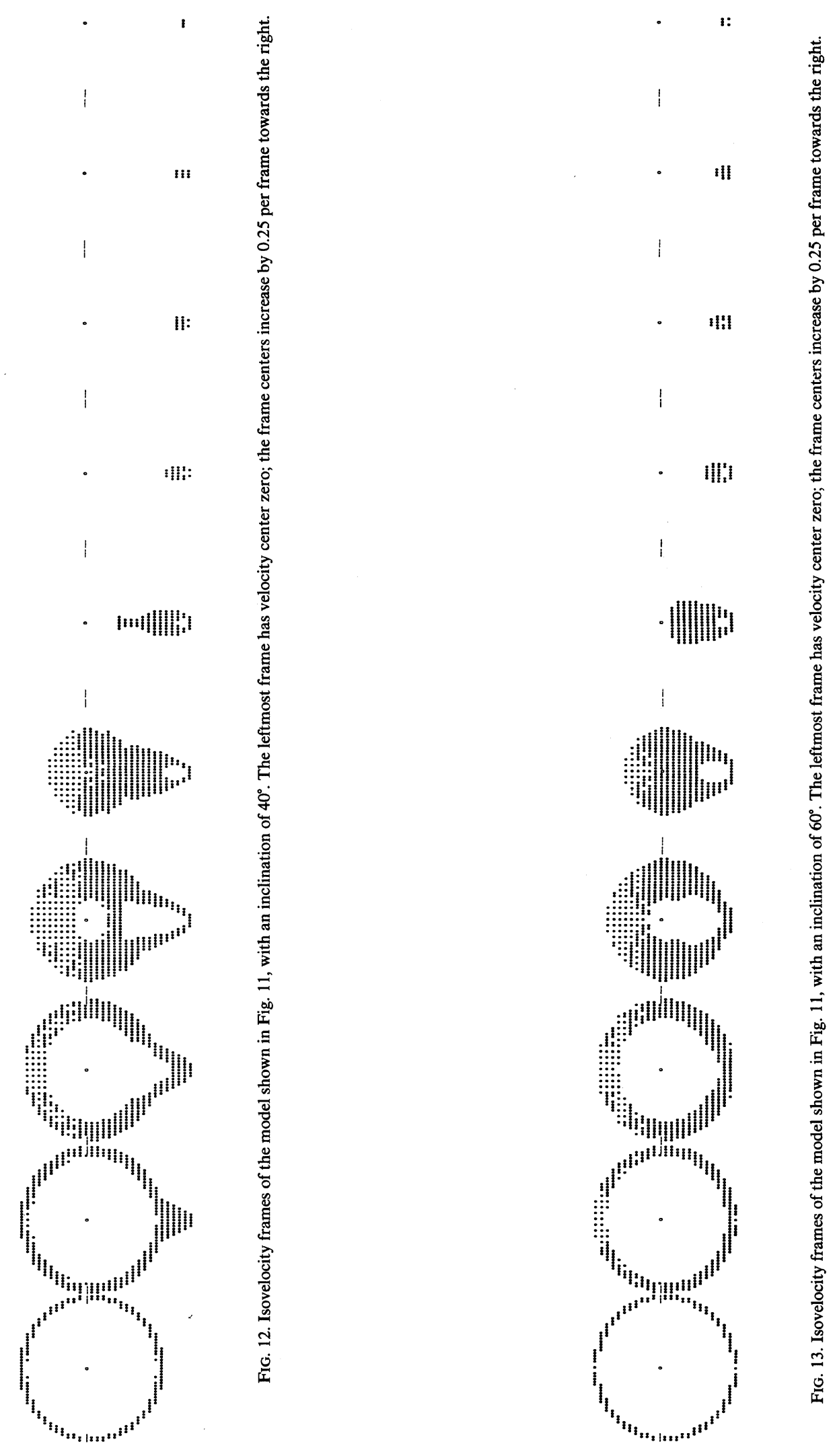




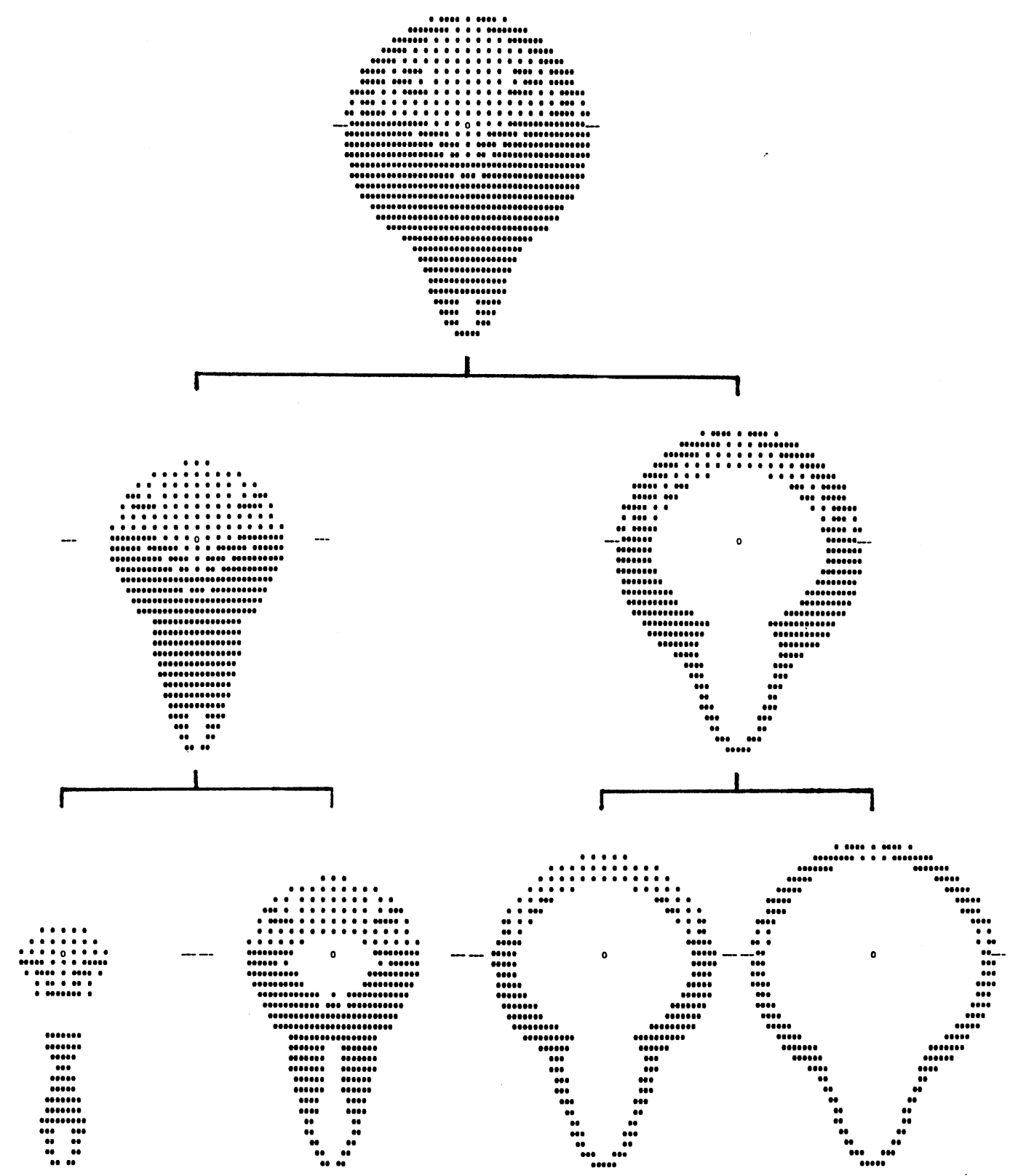

FIG. 14. Isovelocity frames of the model shown in Figs. 11-13, for various velocity centers and channel widths. Top: center 0.75, width 0.8. Middle: centers 0.95 and 0.55 , width 0.4 . Bottom: centers $1.05,0.85,0.65$, and 0.45 , width 0.2 . Note the dramatic effect of increased velocity resolution.

Accordingly, it is premature to interpret the relative brightnesses in our kinematic images in a straightforward way. Still, one ought to expect the denser regions to be brighter because the cooling rate is a quadratic function of the density. This might be relevant for explaining the brightness enhancement of the polar caps. Not only will the density be somewhat higher when the passing bow shock compresses the shell, but also it must be expected that the neutral knots are surrounded by placental gas that has a significantly higher emission measure than that in the equatorial stream.

\section{BROAD APPLICATIONS}

We have developed a hydrodynamic flow model to account for the morphologies and kinematics of $\mathrm{eE}$ and $\mathrm{mE}$ PNs. In this section we explore the successes and shortcomings of the model to these and other classes of PNs.

\section{a) Applicability of the Model to Other PNs}

The observed features of planetary nebulae images that are encompassed by our model are the following. First, the 
presence of a "waistband" in the envelope is our starting point. There is much direct evidence (e.g., Paper I, Kupferman 1983) for equatorial density enhancements in the RGEs of all noncircular PNs.

Second, a stellar fast wind is a necessary ingredient. We concur with most workers in the field that such a wind is very frequently present, even though direct observations thereof (e.g., in the Bremsstrahlung infrared) are difficult. In particular, it would be nice to observe the reverse shock expected in such a wind directly, but its small probable size (Kahn 1983) is an enormous obstacle. Perhaps, if some atomic species in the shock are not completely ionized, wide UV absorption lines seen against the central star will reveal the presence of the inner shock.

Third, the presence of an ellipsoidal outer shock follows from the first two ingredients. The need for such a feature to explain the spectral-line splitting observed throughout planetary nebulae was established early this century (Pottasch 1984 , p. 128), but its explanation as a dynamical consequence of the envelope/wind interaction is recent (Kahn and West 1985; Icke 1987). Circumstantial evidence for the correctness of this view was provided by O'Dell and Ball (1985), who found that the outflow velocity $v$ of the ellipsoid is proportional to its local radius $\mathrm{R}$. Now that is precisely what to expect: In the Kompaneets approximation (cf. Icke 1987), the shock velocity scales as

$$
v \propto \sqrt{P_{0} / \rho_{1}},
$$

Where $P_{0}$ is the pressure in the bubble, and $\rho_{1}$ is the density in the envelope. In an envelope moving with constant speed, we have

$$
\rho_{1} \propto R^{-2}
$$

from which it follows that $v$ is proportional to $R$.

Fourth, we have added the ansae as another consequence of the interaction between the RGE and the wind when the reverse shock geometry is favorable. In particular, we claim that the collimation, the low ionization, and the speed of high-density axial knots follow naturally from hydrodynamic focusing through the reverse shock. The bow shocks around such supersonic knots produce kinematic signatures that correspond well to the ansae and high-velocity patches observed in the velocity images.

Fifth, the polar caps can be seen as a by-product of the axial focusing, in the sense that such focusing is intrinsically imperfect, so that some of the gas near the axis is deflected away from the equator but not enough to contribute to the dense axial stream (see the inner flow lines in Fig. 9). This gas is then responsible for enhancing the polar cap emission by collisions and perhaps forming local shocks.

Spectroscopically, we expect to find that the knots at the tips of the ansae are in a state of low ionization. Near the head of the bow shock, especially in the small stagnation zone directly ahead of the knot, ionization should be collisional, whereas the part of the knot that faces the central star might be photoionized. As expected, direct $\mathrm{CCD} \mathrm{H} \alpha$ pictures usually contain, at most, a faint trace of the polar knots, whereas the [N II] maps show them clearly. Spatial resolution of about 1 " may not allow a clean separation of the front and the rear of the knots, so we must leave our expectation of a distinction between the two as a prediction for future $H S T$ observations.

On the other hand, even mild density stratification can produce highly collimated (if perhaps transient) flows (Icke 1987). It is not implausible to suggest that similar processes may operate in other astrophysical situations that involve fast winds. Collimated flows near pre-main-sequence stars, $\mathrm{x}$-ray stars, certain types of close binaries, and even the bipolar outflows seen emerging from some galactic nuclei may have similar origins.

Finally, we note in closing this section that it would be imprudent to scrap the possibility that the shaping of spindle-type planetaries is due to events around a binary central star-see, for example, Kolesnik and Pilyugin (1986). However, as discussed in Paper I, very few binaries have been found in PNs with highly articulate structure. Typically, close binary PN nuclei are associated with very small, or large and amorphous, nebulae-with the single exception of NGC 6826. Bond and Grauer (1987) estimate that about $10 \%$ of PNs have nuclei with periods less than one day.

\section{b) Model Shortcomings}

The present model assumes stationary flows and hence does not predict the evolution of nonspherical PNs. In addition, the model requires considerable modification if it is to be applied to PNs in which the equatorial-polar contrast is extremely high (so that the equator can be considered a dense physical disk, the outer portions of which may not be heated and ionized by stellar photons). PNs with very dense disks are thought to evolve into the butterfly class of PNs, as discussed in Paper I. Finally, the morphologies of a small minority of PNs are very complex (e.g., NGC 6543) or irregular (e.g., NGC 6210). An adequate description of their geometries for computing models seems impossible. In other words, the present model should be of direct applicability to early- and middle-type PNs only.

Within the appropriate sample of PNs, several questions remain beyond the scope of the model. For example, can the high densities in the knots drive the gas to a low- or neutralionization state? Are irregularities in the outer shock accurately reflected in the geometry of the reverse shock? And, of course, the physical mechanism causing pre-PN nuclei to eject their envelopes with higher densities along an equatorial plane is an important conundrum for which we currently have no solution.

It would be too much to expect this simple model to predict all of the details, even in the present selected sample of PNs, and certainly there are deviations from what we expect. For example, in the kinematic image of NGC 7009, we observe that the caps extend to larger velocity extremes than do the tips of the ansae (cf. Reay and Atherton 1985) and that the symmetry axes of the ansae, on the one hand, and the polar caps, on the other, are not aligned. This can be encompassed in the present model, but only by the ad hoc assumption that the axis of the focused flow wobbles in time. Indeed, evidence of wobble of the twisted bright elliptical rim of NGC 7009 is apparent in its He II $\lambda 4686 \AA$ image (Paper I).

For NGC 7009, the velocity offset between the caps and the ansae is about three isovelocity frames, while these features can be seen over about four frames. This implies a mismatch of $3 / 4$ radian $=40^{\circ}$. It is somewhat worrisome that this value is larger than any found in other nebulae, with the possible exception of NGC 6543. The caps in that nebula are not symmetric about the ansae. Balick and Preston (1987) argue that either precession or episodic gas flows (or collimation), or both, are needed in order to account for the strange morphology of this PN. 
V. CONCLUSIONS

We begin by listing the major observational results of relevance in this paper (and, for didactical purposes, from $\mathbf{P a}$ per I):

(1) Early elliptical (eE) PNs are characterized by a bright prolate spheroidal bubble (seen as a bright elliptical rim) embedded in a smooth halo of amorphous nebulosity, thought to be the former envelope expelled by a precursor red giant. The rim is identified as the shock driven into the RGE by the hot, wind-heated bubble inside. Except when viewed nearly pole-on, the halo shows enhanced emission measure along the minor axis of the spheroid (by factors of about 2 before correction for inclination). Middle elliptical $(\mathrm{mE})$ PNs are generally similar to eEs, except that the ends of the prolate spheroid are truncated where they reach the outer edge of the RGE.

(2) Most eE types show low-ionization knots lying along the extension of lines drawn from the PN nucleus through segments of high curvature in the bright rim (i.e., through the tips of the spheroid). The knots come in pairs on nearly opposite sides of the nucleus, and are similarly symmetric in velocity space. The knots appear to be connected to the rim nearby by triangular (conical?) patches of high-ionization gas. The knots and the emission patches are called "ansae" in this paper.

(3) The bright spheroidal rim expands systematically and, so far as we can determine, uniformly. The nonuniform velocity that occurs in our shock-propagation model is hard to detect in eE types, since the expected differences in the isovelocity frames amount to only $5 \%-10 \%$. We will address this point in a forthcoming publication on butterflytype PNs. In the present sample of $\mathrm{eE}$ and $\mathrm{mE}$ PNs, the spheroid is expanding into the RGE; indeed, Chu and Jacoby (1987) find that the ellipsoid expands into the halo in all PNs they observed (except IC 3568).

(4) Knots are characterized by observed velocities of 20 $170 \mathrm{~km} \mathrm{~s}^{-1}$. Based on our model (summarized below), the correction for projection effects can be large, and the true space velocities are likely to exceed $\sim 60 \mathrm{~km} \mathrm{~s}^{-1}$. The triangular patches of nebulosity are also part of a collimated radial outflow of lesser observed velocity than the knots.

(5) In mE PNs, knots and filaments protrude outside the RGE from the open ends of the bright rims. The ensemble of knots and filaments appear to form a pair of small conical surfaces that extend from the knots backward to the open ends of the spheroid.

(6) Two PNs in the present sample, NGC 6543 and 7009, show caps of low ionization inside the ends of the long axis of their halos. Like the knots, the caps have no $\mathrm{H} \alpha$ or [O III] counterparts (Paper I). Gas kinematics do not follow the general patterns of motions observed in nearby regions of the spheroid, halo, or knots.

We propose a model that accounts for both the morphologies and the kinematics of $\mathrm{eE}$ and $\mathrm{mE}$ PNs. The model requires (1) a fast stellar wind (like those observed in the nuclei of PNs), presumed isotropic, and (2) an inner halo (RGE) in which the densities are slightly larger along an equatorial plane than toward the poles (as observed). Hence the stellar wind encounters more resistance (possibly as much as $50 \%$ in butterfly types) in the equatorial zones.

Due to the nonaxisymmetric density distribution in the $\mathrm{RGE}$, the wind drives a shock of prolate elllipsoidal geometry into the envelope. The major axis of this shock coincides with the polar axis of the inner halo. An invisible prolate reverse shock width of the same orientation as the outer ellipsoid drives towards the star through the hot windheated interior of the ellipsoid. Matter flowing from the star is deflected away from the shock normal as the gas passes through the prolate inner shock. In regions where the reverse shock makes the largest angle with the radial direction (namely near the tips of the spheroid), the streamlines can converge, and the flow can become collimated (Icke 1987).

Assuming that cooling processes in the collimated flow become effective once the stream leaves the hot-bubble interior, then thermal instabilities will develop in the densest part of the collimated flow. Typically, this will occur on opposite sides of the central star and produce a bipolar symmetry. Because the cooling rate is a very strong function of temperature- $\exp (-1 / T)$ for forbidden lines and $T^{3-4}$ for dust - the densities in the cool knots will become even larger, and hence the knots will have larger recombination rates and lower ionization stages than their surroundings. Outwardmoving, low-ionization knots are found in NGC 3242, 6826, 7662 , and, perhaps, in NGC 2392, which is probably observed close to its polar axis. The small, triangular patches of high-velocity gas behind the knots can be identified with the bow shock that connects the knots with the rim behind them.

If the gas stream near the knots contacts the RGE, and if this less-focused gas cools sufficiently fast to maintain its density above that of the general bubble interior, then collisionally heated polar caps of the type seen in NGC 6543 and 7009 will form.

Since the collimated flows have large outward velocities compared to the RGE, they eventually breach the edge of the latter. At this time, the morphology of the PN enters the $\mathrm{mE}$ class. The collimated flows, now only weakly constrained, expand nearly adiabatically into the interstellar medium outside the RGE. The PNs in the sample presented here are too early type to show a well-developed case of this behavior; the hollow cones that are evident in the isovelocity frames of PNs like NGC 7354 are very well reproduced by the velocity field of bow shocks trailing behind the knots (cf. Fig. 11).

Future research is heading in several different directions. First, we plan to observe other $\mathrm{eE}$ and $\mathrm{mE}$ PNs to verify that the model has broad applicability within these classes of PNs. Second, similar imaging and kinematic studies have been obtained for PNs in neighboring morphological classes (early- and middle-type butterfly PNs) in order to determine whether similar physical processes are able to account for their structure and evolution. The analysis of these data and the attendant theoretical modeling are in progress. Third, long-slit spectrophotometric studies of the bright rims, knots, and halos will permit us to specify the density, ionization, and temperature variations within elliptical PNs quantitatively. This will permit us to better specify the halo parameters and the shock-jump conditions for future model calculations.

On the theoretical front, V.I. and H.L.P. are developing a two-dimensional numerical hydrodynamic code that permits time-dependent states of model PNs to be simulated. The conditions for the formation of knots and collimated flows can be studied without recourse to some of the simplifying assumptions made in this paper. These hydrodynamic studies can also be applied to other classes of astrophysical objects, such as pre-main-sequence stars and active galactic nuclei, in which a detailed description of the environment of the collimated flow is impeded by heavy foreground obscuration or poor spatial resolution. 
It is a sincere pleasure to thank the staff of the Kitt Peak National Observatory for their enthusiasm and interest in obtaining useful observations. The generous advice of George Jacoby and Caty Pilachowski are especially appreciated. You-Hua Chu has graciously supplied us with important information in advance of publication. Financial support for B.B. and H.L.P. was received from NSF grants
82-08041 and 86-12228, and V.I. gratefully acknowledges travel support from the Leids Kerkhoven-Bosscha Fonds. All image analysis was performed at the Astronomical Image Processing Facility of the University of Washington. Equipment procurement was made possible by financial support from the NSF (AST 83-10552) and the Digital Equipment Corporation.
Balick, B. (1987a). Sky and Telesc. 73, 125.

Balick, B. (1987b). Astron. J. 94, 671 (Paper I).

Balick, B., Bignell, C. R., Hjellming, R. M., and Owen, R. (1987). Astron

J. 94, 948.

Balick B., and Preston, H. L. (1987). Astron. J. 94, 958.

Bond, H.E., and Grauer, A. D. (1987). Space Telesc. Sci. Inst. Prepr. No 182.

Castor, J., McCray, R., and Weaver, R. (1975). Astrophys. J. Lett. 200, L107.

Courant, R., and Friedrichs, K. O. (1976). Supersonic Flow and Shock Waves (Springer, Berlin).

Field, G. B., Goldsmith, D. W., and Habing, H. J. (1969). Astrophys. J. Lett. 155, L149.

Friend, D. G., and Abbott, D. C. (1986). Astrophys. J. 311, 701.

Gieseking, F., Becker, I., and Solf, J. (1985). Astrophys. J. Lett. 295, L17.

Icke, V. (1987). Astron. Astrophys.( to be published).

Jacoby, G. H. Quigley, R. J., and Africano, J. L. (1987). Publ. Astron. Soc. Pac. 99, 672.

Kahn, F. D. (1983). In Planetary Nebulae, IAU Symposium No. 103, edited by D. R. Flower (Reidel, Dordrecht), p. 305.

Kahn, F. D., and West, K. A. (1985). Mon. Not. R. Astron. Soc. 212, 837. Kolesnik, I. G., and Pilyugin, L. S. (1986). Astron. Zh. 63, 279.

Kupferman, P. N. (1983). Astrophys. J. 266, 689.

Kwok, S. (1980). J. R. Astron. Soc. Can. 74, 216.

Kwok, S. (1982). Astrophys. J. 258, 280.

Kwok, S., Purton, C. R., Matthews, H. E., and Spoelstra, T.A.T. (1985).

\section{REFERENCES}

Astron. Astrophys. 144, 321.

Lamers, H. J. G. L. M. (1983). In Diffuse Matter in Galaxies, edited by J. Audouze (Reidel, Dordrecht), p. 35.

Landau, L. D., and Lifschitz, E. M. (1959). Course in Theoretical Physics VI: Hydrodynamics (Pergamon, London).

Mathews, W. G. (1978). In Planetary Nebulae, Observations and Theory, edited by Y. Terzian (Reidel, Dordrecht), p. 251.

O'Dell, C. R., and Ball, M. E. (1985). Astrophys. J. 289, 526.

Okorokov, V. A., Shustov, B. M., Tutukov, A. V., and Yorke, H. W. (1985). Astron. Astrophys. 142, 441.

Phillips, J. P., and Reay, N. K. (1983). Astron. Astrophys. 117, 33.

Pottasch, S. R. (1984). Planetary Nebulae (Reidel, Dordrecht), p. 175.

Reay, N. K., and Atherton, P.D. (1985). Mon. Not. R. Astron. Soc. 215, 233.

Reay, N. K., Atherton, P. D., and Taylor, K. (1983). Mon. Not. R. Astron Soc. 203, 1087.

Reay, N. K., and Worswick, S. P. (1982). Mon. Not. R. Astron. Soc. 199, 581.

Sabbadin, F., and Hamzaoglu, E. (1982). Astron. Astrophys. 109, 131.

Solf, J. (1986). Mitt. Astron. Ges. 69, 166.

Van Dyke, M., editor (1982). An Album of Fluid Motion (Parabolic, Stanford, CA).

Volk, K. M., and Kwok, S. (1985). Astron. Astrophys. 153, 79.

Weaver, J., McCray, R., Castor, J., Shapiro, P., and Moore, R. (1977). Astrophys. J. 218, 377.

Weedman, D. W. (1968). Astrophys. J. 153, 49. 


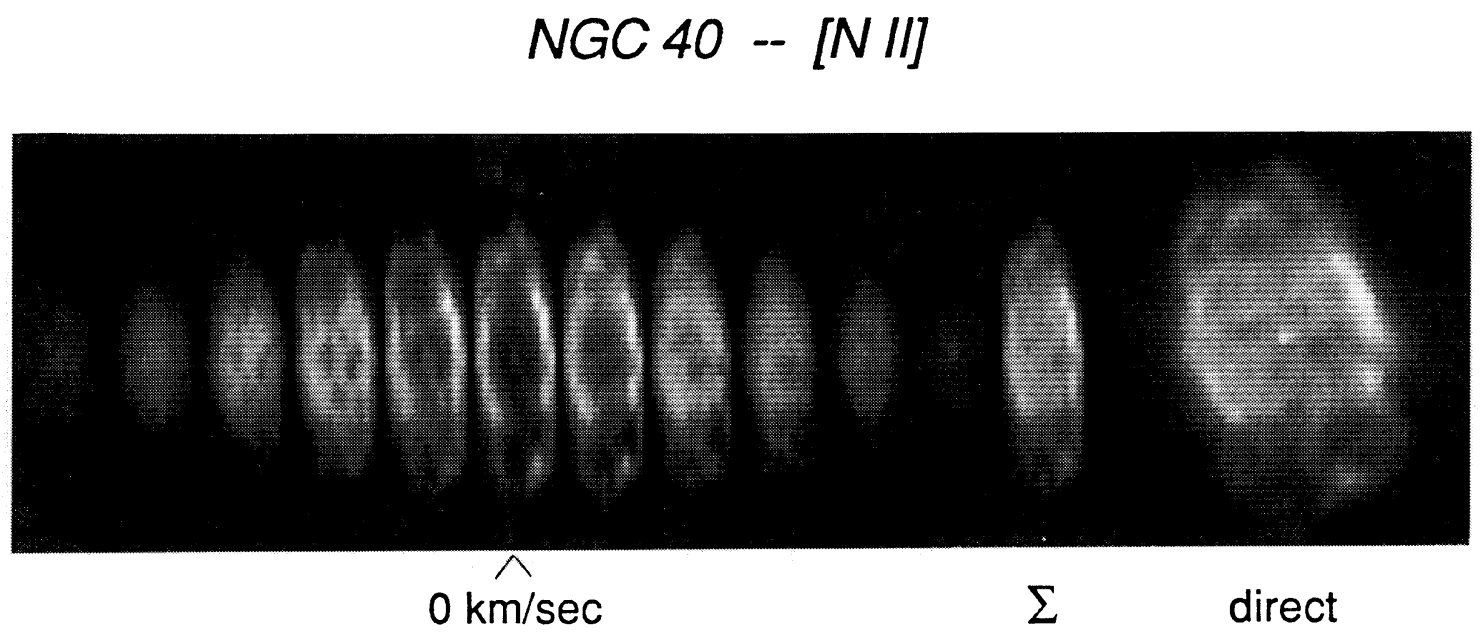

FIG. 1. Kinematic image of NGC 40 in the line of [N II]. The first 11 frames show the nebula as it would appear through narrowband filters of width and relative offsets of $8.91 \mathrm{~km} \mathrm{~s}^{-1}$. The sixth frame corresponds to zero velocity. North is up in all frames. The pixel sizes on the sky are given in Table I. These frames have been added to produce the 12th, or sum, frame, which shows the nebula as it would appear through a filter that included all the line emission. The final frame is an undistorted direct CCD image in the [N II] line from Paper I for comparison (see Paper I for details ).

Balick et al. (see page 1642) 


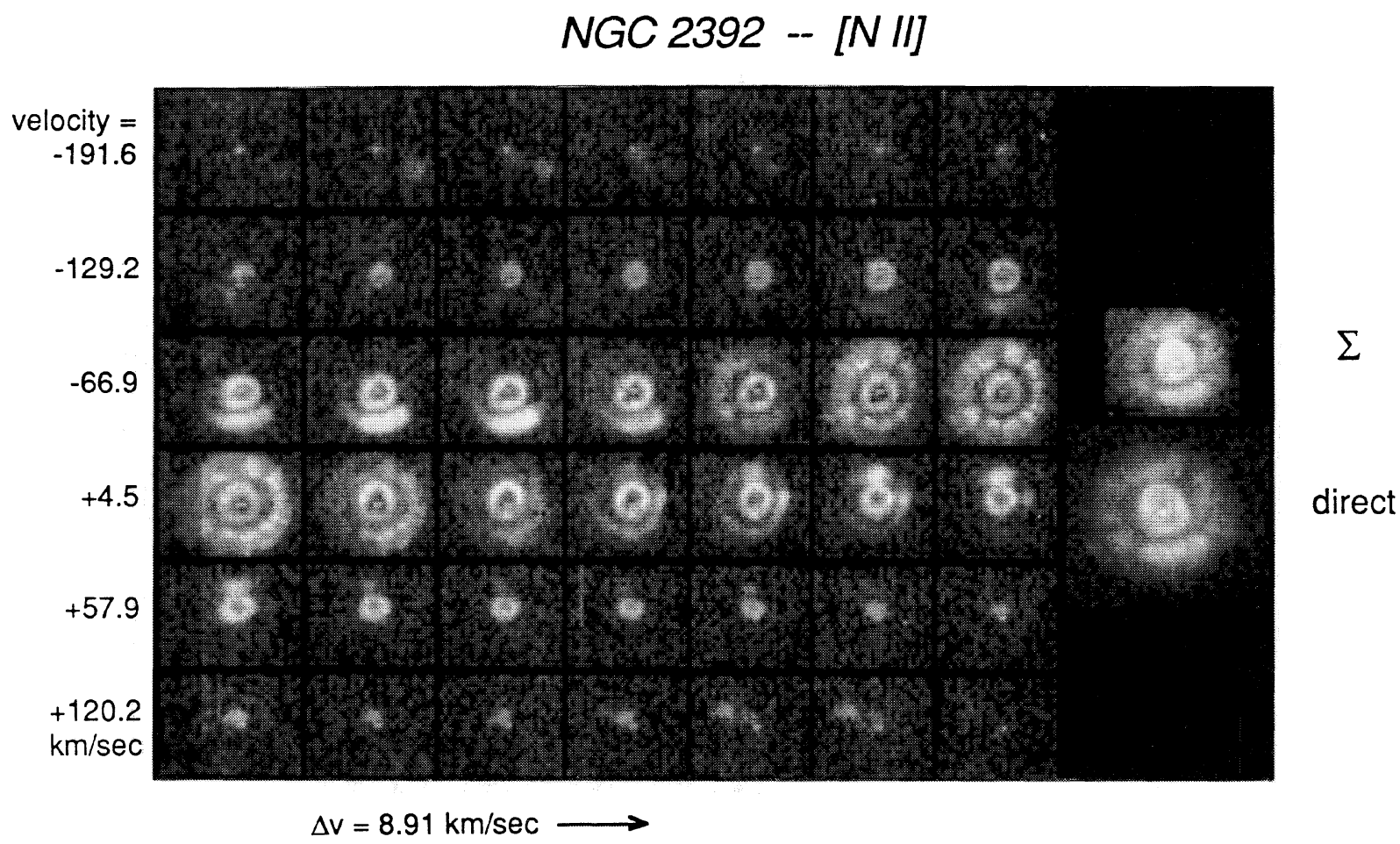

FIG. 2. Kinematic image of NGC 2392 in the line of [N II]. Frames increase in velocity to the right and down the page. Zero velocity lies between frames 21 and 22. The sum frame is shown in the upper frame on the far right, and the direct CCD frame is beneath it. See also Table $I$ and the caption of Fig. 1. N is up in all frames.

Balick et al. (see page 1642) 


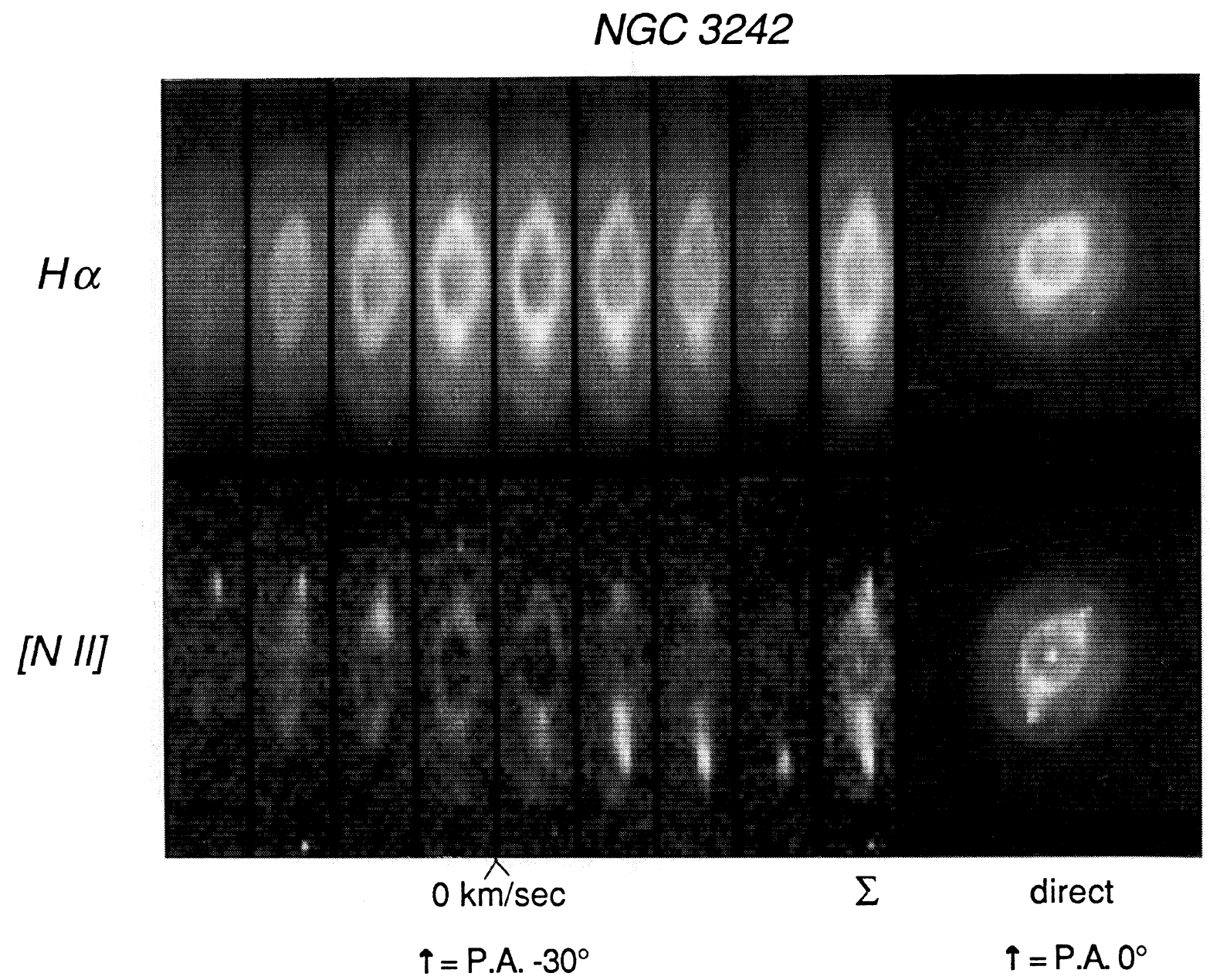

FIG. 3. Kinematic images of NGC 3242 in the lines of $\mathrm{H} \alpha$ (top row) and [N II] (bottom row). The format of the figure is described in Table I and in the caption of Fig. 1. Zero velocity lies between frames 4 and 5 in each row. The vertical direction in the kinematic images corresponds to a vector in P.A. $-30^{\circ}$ in the direct $\mathrm{CCD}$ image (in which $\mathrm{N}$ is up)

Balick et al. (see page 1642) 


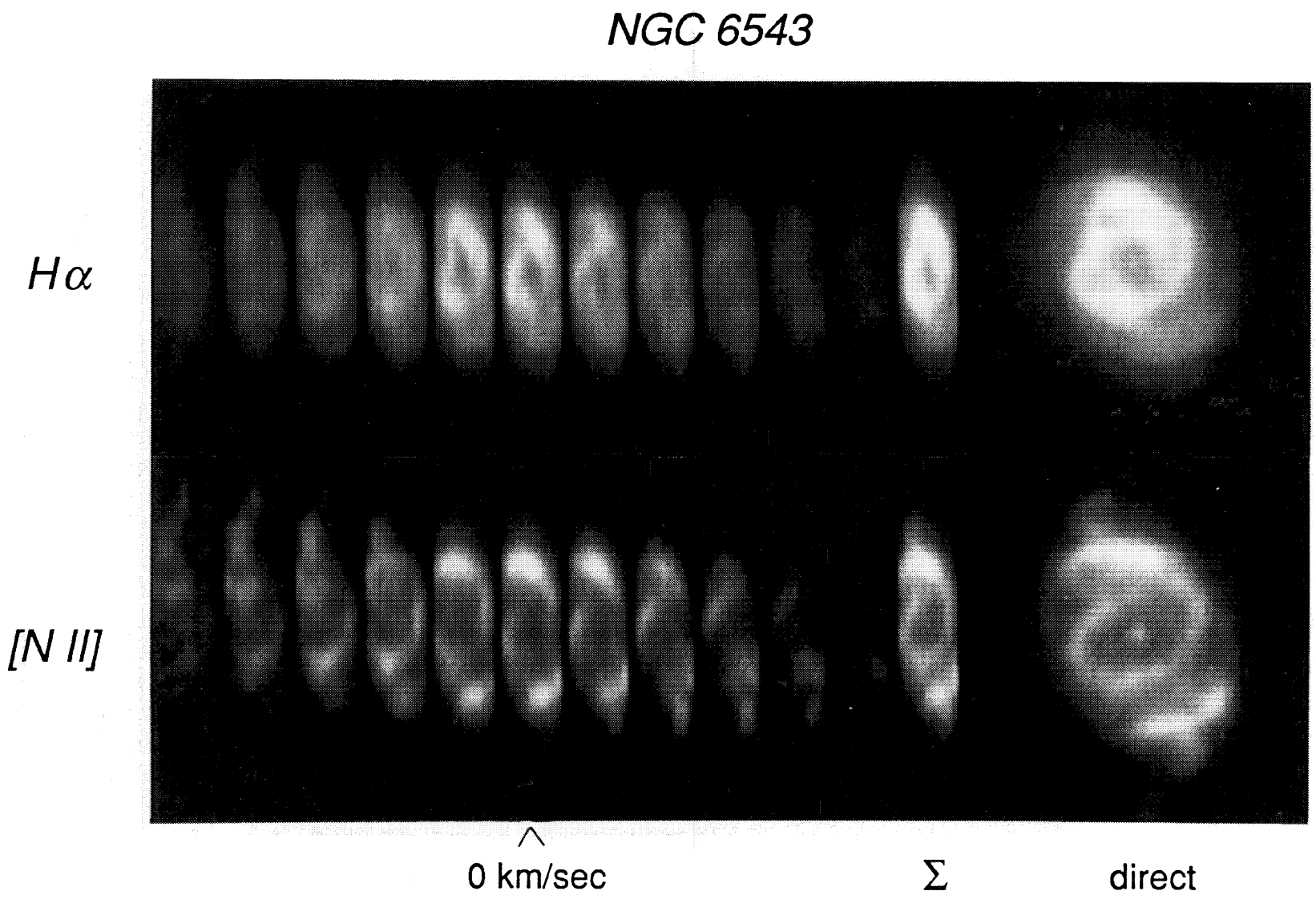

Fig. 4. Kinematic images of NGC 6543 in the lines of $\mathrm{H} \alpha$ (top row) and [N II] (bottom row). The format of the figure is described in Table I and in the caption of Fig. 1. Zero velocity corresponds to frame 6 in each row. $\mathrm{N}$ is up in all frames.

Balick et al. (see page 1642) 


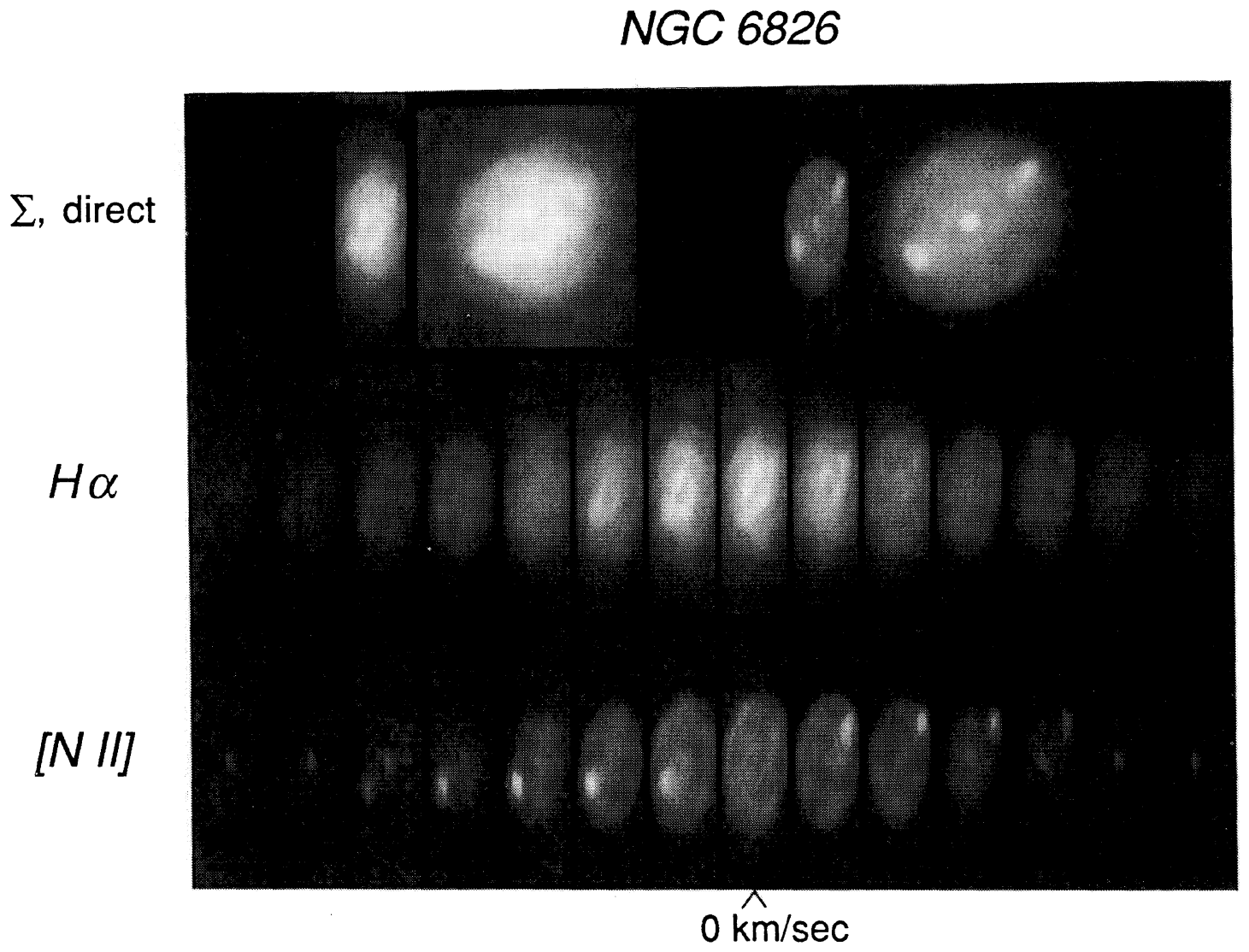

FIG. 5. Kinematic images of NGC 6826 in the lines of $\mathrm{H} \alpha$ (middle row) and [N II] (bottom row). The format of the figure is described in the caption of Fig. 1. Zero velocity corresponds to frame 8 in each of these rows. Across the top row are, respectively, the sum and direct $\mathrm{CCD}$ frames in $\mathrm{H} \alpha$ and the sum and direct frames in [N II]. See Table I and caption of Fig. 1 for further details. N is up in all frames.

Balick et al. (see page 1642) 


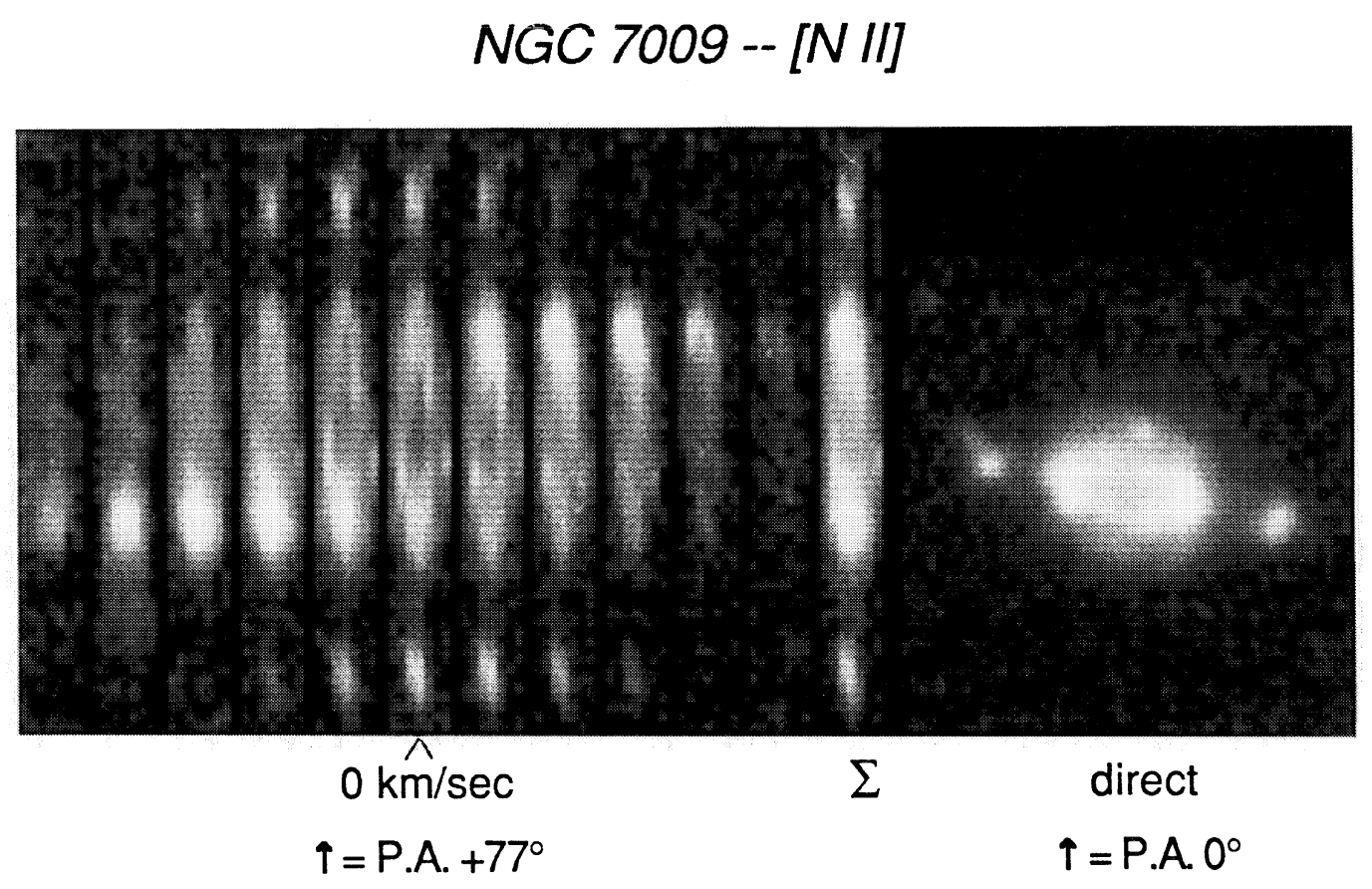

FIG. 6. Kinematic images of NGC 7009 in the line of [N II]. The format of the figure is the same as in Fig. 1. See also Table I. The vertical direction in the kinematic images corresponds to a vector in P.A. $+77^{\circ}$ in the direct CCD image (in which $\mathrm{N}$ is up).

Balick et al. (see page 1642) 


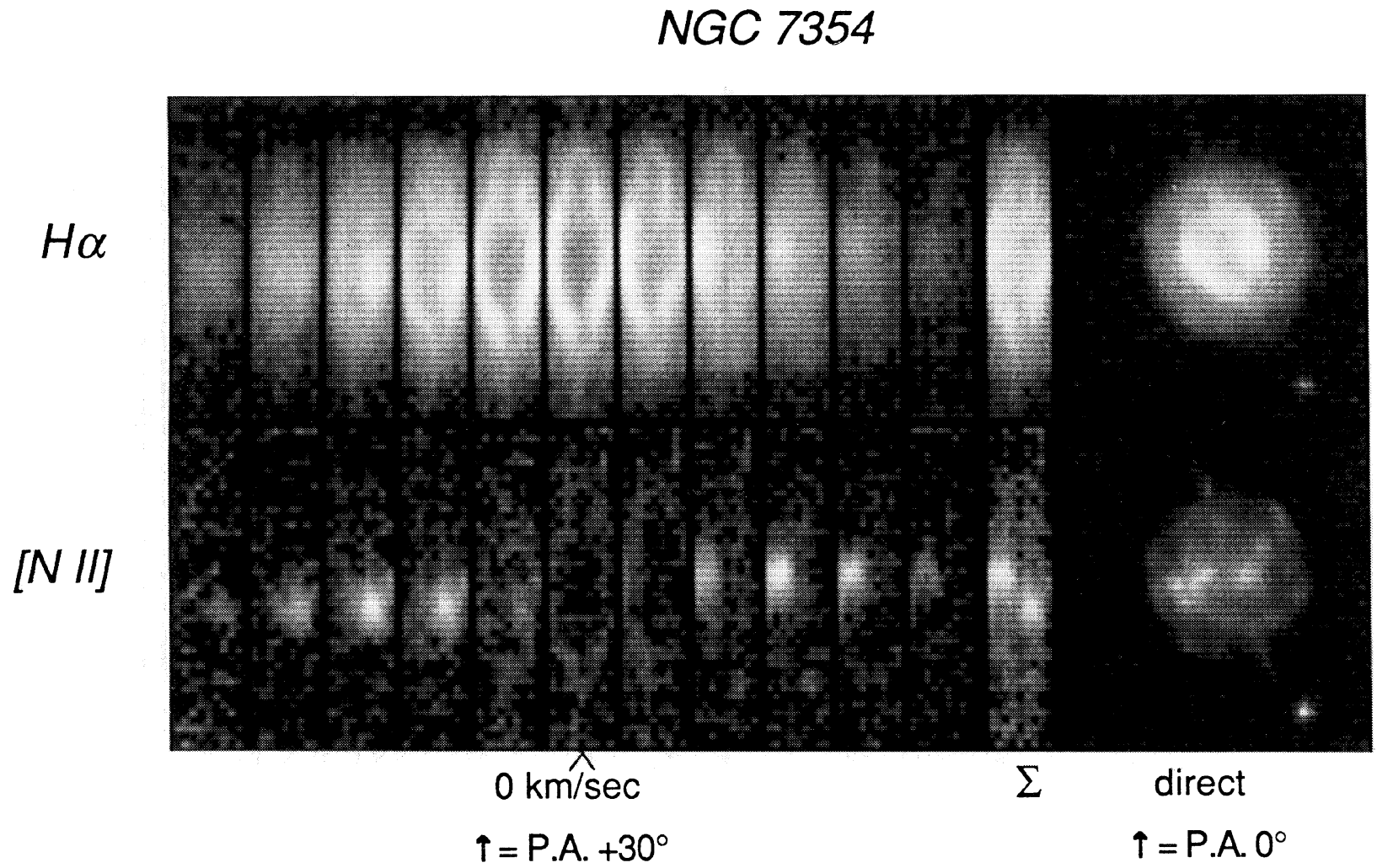

FIG. 7. Kinematic images of NGC 7354. The format is the same as in Fig. 4. The vertical direction in the kinematic images corresponds to a vector in P. A. $+30^{\circ}$ in the direct CCD image (in which $\mathrm{N}$ is up). See also Table $\mathrm{I}$.

Balick et al. (see page 1642) 


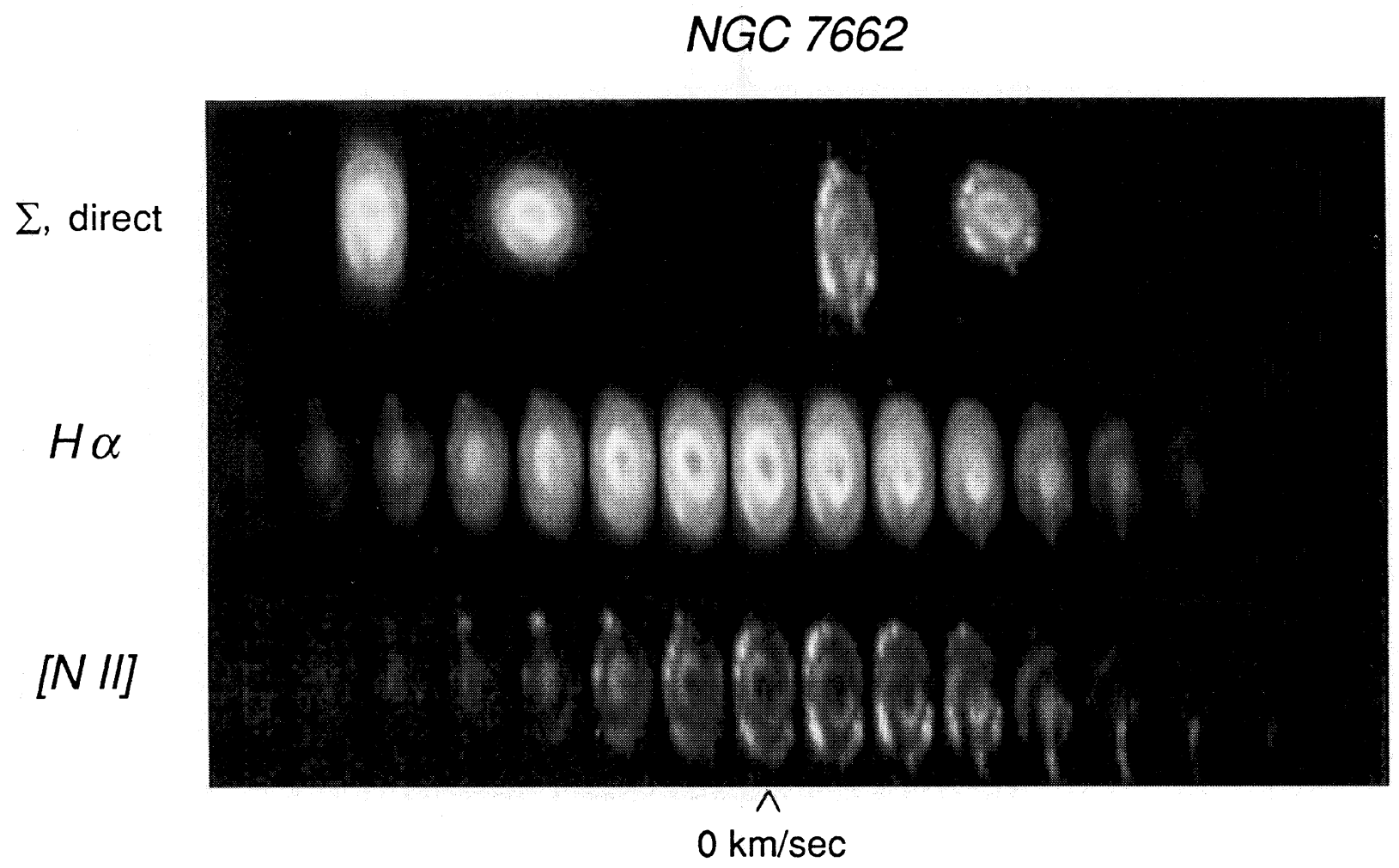

FIG. 8. Kinematic images of NGC 7662. The format is the same as in Fig. $5 . \mathrm{N}$ is up in all frames. See also Table I.

Balick et al. (see page 1642) 